\title{
Risk Factors for Smear Negative And Culture Positive Results Among Pulmonary Tuberculosis Patients in Mwanza, Tanzania
}

\author{
Range Nyagosya ${ }^{1, *}$, Åse B. Andersen ${ }^{2}$, Frank van Leth ${ }^{3,7}$, Pascal Magnussen ${ }^{4}$, \\ Mugomela Apolinary ${ }^{5}$ and Henrik Friis ${ }^{6}$
}

${ }^{I}$ NIMR, Muhimbili Medical Research Centre, Dar es Salaam, Tanzania; ${ }^{2}$ Department of Infectious Diseases, Rigshospitalet, Denmark; ${ }^{3}$ KNCV Tuberculosis Foundation, The Hague, The Netherlands; ${ }^{4}$ DBL-Centre for Health Research and Development, Charlottenlund, Denmark; ${ }^{5}$ Zonal TB Reference Laboratory, Mwanza Tanzania; ${ }^{6}$ Department of Human Nutrition, University of Copenhagen, Denmark and ${ }^{7}$ Centre for infection and Immunity, Academic Medical Centre, University of Amsterdam, The Netherlands

\begin{abstract}
Background: Smear negative and culture positive results in pulmonary tuberculosis are not uncommon yet the underlying factors are not well established.

Objective: To determine factors associated with smear negative and culture positive results.

Methods: Pulmonary tuberculosis patients were consecutively recruited for 12 months from five health facilities in Mwanza region, Tanzania. Sputum examinations were done at the recruitment centre and at the TB reference laboratory using Auramine $\mathrm{O}$ technique. Culture was done at the TB reference laboratory using Lowenstein Jensen solid media. A post-hoc analysis compared patients who had a smear negative culture positive result (case) with patients who had not (controls).

Results: A total of 655 pulmonary TB patients were recruited, 18 had no culture results and were excluded from the analysis. Of the remaining 637 patients, $127(19.9 \%)$ had three negative smears at the recruitment centre and 34 patients were a case. Current smoking was strongly associated with being a case, especially in women. Of the 127 patients who had three negative smears at the recruitment center, $104(81.9 \%)$ also had a negative smear at the reference laboratory. Of these, 13 $(12.5 \%)$ were still culture positive.

Conclusion: The frequencies of smear-negative culture-positive results differ between health facilities, indicating possible difference in quality of laboratory procedures. Strengthening of laboratory capacity is needed both for optimizing smear microscopy techniques, and for performing sputum cultures for diagnosing TB when there is a high rate of suspicion. The association between smoking and smear negative culture positive TB needs to be assessed in adequately large studies.
\end{abstract}

Key Words: Pulmonary TB, Smear, Culture, Smoking, Tanzania.

\section{INTRODUCTION}

Tuberculosis (TB) remains one of the deadliest diseases worldwide, with an estimated 8.4 million new cases and over 2 million deaths each year [1]. For decades, smear microscopy has remained the corner -stone for diagnosis of pulmonary tuberculosis (PTB). It is the most feasible microbiological method for the diagnosis of PTB in developing countries due to its rapidity, low cost, and high positive predictive value for Mycobacterium tuberculosis [2]. Sputum smear microscopy with Ziehl-Neelsen staining is only 60 to $70 \%$ sensitive for the diagnosis of pulmonary TB compared with sputum culture [3, 4]. However, sputum cultures need a good laboratory infrastructure; they are expensive and take up to eight weeks to get conclusive results.

Studies conducted in different settings have reported an increasing proportion of $\mathrm{TB}$ patients with negative smear

*Address correspondence to this author at the National Institute for Medical Research (NIMR), Muhimbili Medical Research Centre, P. O. Box 3436, Dar es Salaam, Tanzania; Tel: +255 22 2152232; Fax: +255 222150458 or +255 22 2124500; E-mail: hn_range@yahoo.co.uk results but positive culture results. Known factors to influence the results of either smear microscopy or sputum culture include; HIV infection [5], tuberculin test [6], and smoking [7]. However, data on factors associated with an increased risk of smear negative and culture positive results are scarce.

In Tanzania, sputum culture is not performed for routine diagnosis but mainly for monitoring of TB treatment. With a high prevalence of HIV-infection in the general population $(8 \%)$, there is the potential that a substantial number of TBpatients will be smear-negative. Considering the relatively low sensitivity of smear microscopy it is possible that a substantial number of TB patients may be missed to be initiated on treatment. Without proper treatment, they may continue suffering from the disease and others may ultimately die. Untreated TB contributes to ongoing transmission $[8,9]$.

We conducted post-hoc analysis to determine factors associate with smear negative and culture positive results among PTB patients at Mwanza Tanzania. 


\section{MATERIALS AND METHODOLOGY}

\section{Study Design}

The original study was a randomized, placebo-controlled trial, using a two-by-two factorial design, to assess the effects of daily supplementation with a multi-micronutrient and a zinc tablet throughout the eight months of short course TB treatment (Range TMIH 2005) [10]. The current study is post-hoc analysis using original data.

\section{Population and Study Area}

The 655 TB patients were consecutively recruited from the general out-patient department and from the in-patients from five recruitment health facilities between August 2001 and July 2002. The original randomized study included all smear-positive pulmonary TB $(n=510)$ patients identified at the five recruitment centres in the same period. The patients were recruited based on the study inclusion criteria which included; newly diagnosed pulmonary TB (PTB) patients, aged 15 years or above, willingness to participate in the study, and residents of Mwanza city or Magu district in Mwanza, Tanzania. TB patients with the following criteria were excluded; TB patients registered as return to control or smear positive failure cases; patients with clinical evidence of renal, hepatic or cardiovascular disease; pregnant or lactating women; patients with advanced respiratory or other diseases considered unlikely to survive. For the current analysis we added the data of the TB patients who were identified at the same institutions and in the same time frame, but they did not meet the inclusion criteria of being smear-positive $(n=127)$. The 127 local smear pulmonary cases were not randomized but rather included as controls for the different variables assessed among randomised cases for comparison of other parameters that were of interest and were assessed in the study primary objective. The variables include; HIV- infection, parasitic infections, body composition, etc. Given the passive case finding of the study subjects, and the identical participating health facilities and time of recruitment, the total population for this study can be regarded as homogeneous.

Patients who were suspected of having TB were confirmed through clinical examination, smear microscopy, culture and or chest $\mathrm{x}$-ray. All identified patients at the recruitment centres were requested to submit an early morning sputum specimen in a universal bottle for subsequent smear microscopy and culture at the Zonal TB reference laboratory (ZTRL). The early morning sputum specimen that was sent to the TB reference laboratory was collected on the second day after the start of TB treatment. The sputum was collected before the second dose of anti-TB drugs was swallowed. Smear microscopy at the recruitment centres was done using Ziehl-Neelsen staining technique [11], while at the ZTRL, fluorochrome (Auramine O) staining technique was used. Culture was done on Lowenstein Jensen (LJ) solid media $[12,13]$. Patients were given information about the study and offered inclusion after informed consent. The study was conducted within the framework of the National TB and Leprosy Programme [14], with diagnosis, classification, registration and treatment of TB patients done in accordance with recommended standard procedures [15].
All confirmed PTB patients at the recruitment centres were started on TB treatment. Prior to starting treatment, the TB Clinical Officers collected demographic and medical history data from all patients. A 10-ml sample of venous blood was collected from each patient and used to check for malarial parasitemia and HIV status, white blood cell counts, including CD4 and CD8 and viral load. Questionnaires were administered to each patient to elicit relevant information on age, marital status, tribe, religion, occupation, smoking habits and alcohol intake [16].

\section{Ethical Consideration}

Permission to conduct the study was granted from the Ethics Committee of the National Institute for Medical Research (NIMR) in Tanzania, and the Danish National Committee on Biomedical Research Ethics recommended the study. Permission was also obtained from the Regional Medical Officer and the Council Medical Officers in Mwanza Region. Informed oral consent was obtained from all study participants. Pre-test HIV counselling was given to all, and post-test counselling was given to those who were interested to know their HIV results.

\section{Statistical Analysis}

For the current post-hoc analysis we compared the characteristics of the patients who were smear negative but culture positive (case) with the patients who were not (control). A case was defined as a patient with three consecutive negative $\mathrm{ZN}$ microscopy smears at the recruitment center, and a positive culture at the reference laboratory. Patients who did not have a culture performed or whose culture was contaminated were excluded form the analysis.

Characteristics of the patients in the two groups were compared with Fisher's exact test for categorical variables and Kruskall-Wallis test for continuous variables.

Factors independently associated with being a case were assessed through unconditional logistic regression. The different logistic models were tested for significance with the $\log$-likelihood test. However, variables could be kept into the model despite a non-significant log-likelihood test when this improved the interpretation of the data. All logistic models included sex and age (continuous) as possible confounding variables.

Given the possibility of a differential performance of laboratory staff at the centers, we adjusted the standard errors (use of a robust standard error) around the point estimates for possible clustering of the data within a center by adding the variable 'center' as a clustering variable. The level of statistical significance was set at $5 \%$. The analyses were performed using STATA version 9.2 (StataCorp. Texas USA).

\section{RESULTS}

The study included a total of 655 patients from five different health facilities. Of these, 18 did not have an appropriate culture result and were excluded from the analysis (Fig. 1). Of the 637 patients remaining, 127 (19.9\%) had three negative $\mathrm{ZN}$ microscopy smear slides at the recruitment centre (Table 1). Of these, $34(26.8 \%)$ had a positive culture in 


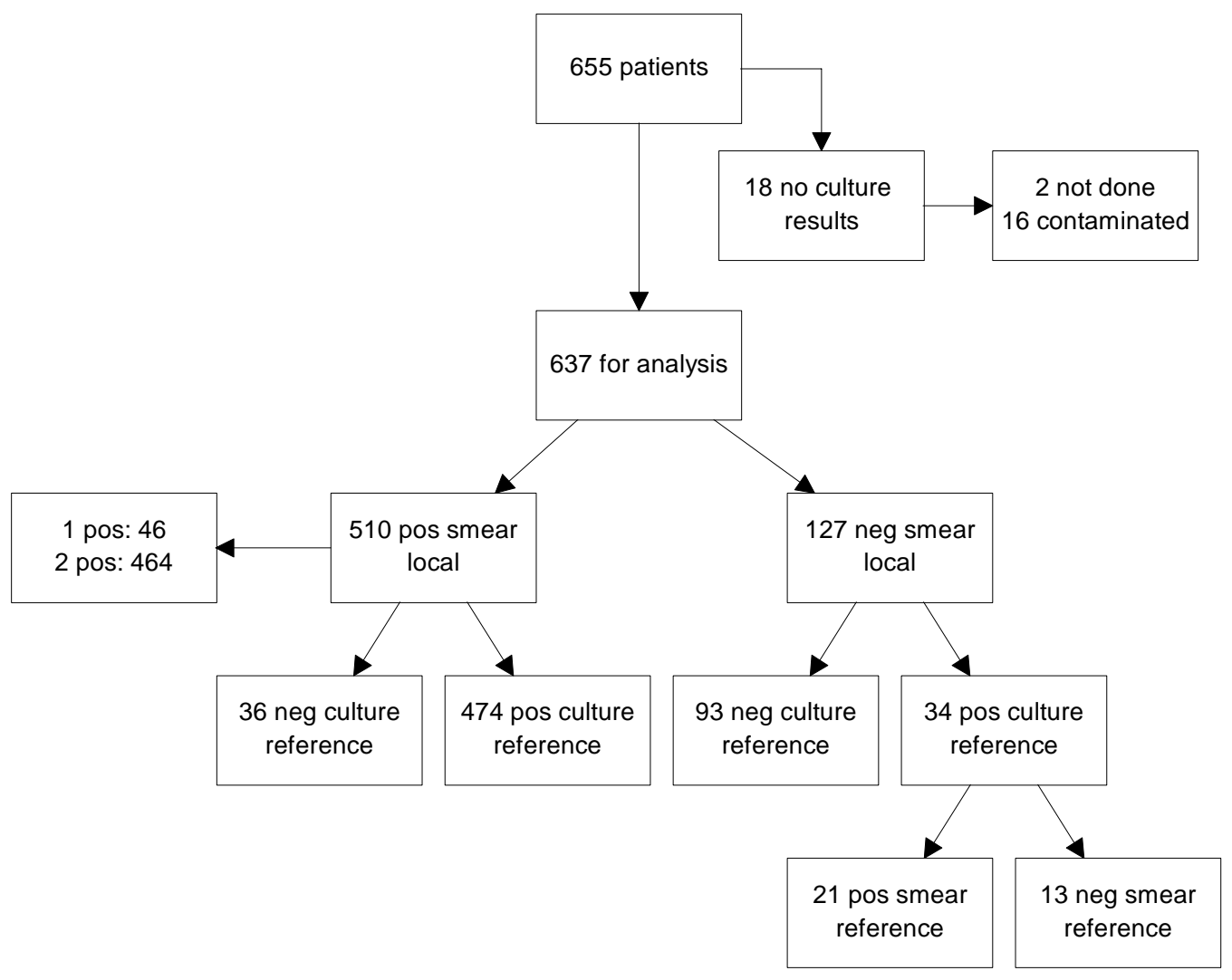

Fig. (1). Patient deposition.

the reference laboratory and were considered a case for this study, all other patients were considered a control.

Table 1. Distribution of Smear-Negative Patients and Cases by Diagnostic Centers

\begin{tabular}{|c|c|c|c|}
\hline & Patients & Smear-Negative (\%) & Cases (\%) \\
\hline \hline Sekou Toure & 290 & $36(12.4)$ & $21(7.2)$ \\
\hline Bugando & 136 & $54(39.7)$ & $8(5.9)$ \\
\hline Magu & 109 & $19(17.4)$ & $3(2.8)$ \\
\hline Buzuruga & 63 & $14(22.2)$ & $0(0.0)$ \\
\hline Butimba & 39 & $4(10.3)$ & $2(5.1)$ \\
\hline
\end{tabular}

The characteristics of the two groups of patients are summarized in Table 2. The number of cases differed per diagnostic center with relatively more cases in Sekou Toure, and relatively less cases in Magu and Buzuruga (no cases at all). The HIV-status of the patients did not differ statistically significant between the two groups but was numerically higher in the cases. The severity of the immunosuppression of HIV-positive patients was comparable between cases and controls based on CD4+ cell counts. Nor did the body weight or body mass index differ across the group. The only statistically significant difference between the two groups was the percentage of the patients who were current smokers. This was $26.5 \%$ for the cases and $11.9 \%$ for the controls ( $\mathrm{p}=$ $0.029)$.
From the variables reported in Table 2 we included current smoking, current alcohol use, and HIV-status in subsequent logistic regression model in addition to sex and age (Table 3). Only current smoking was identified as being associated with being a case (OR: $3.61 ; 95 \%$ Confidence interval $[\mathrm{CI}]: 1.57-8.33)$. Given the differential smoking habits in the general population between men and women, we tested whether there was an interaction between sex and current smoking. The interaction term was indeed strong (OR: 0.16 ; $95 \%$ CI: $0.28-1.00$ ), indicating a stronger association for smoking women to be a case as compared to smoking men. Compared to non-smoking patients, the OR for smoking men of being a case was 2.50 (95\% CI: $1.38-4.51$ ), while it was 17.75 for women (95\% CI: $3.04-103.47)$. The reason for these wide confidence intervals is that there were only 5 women who smoked, out of them $2(40 \%)$ were a case, compared to 76 men who smoked out of them 7 (9\%) were a case.

There was no association between past smoking and being a case (OR 1.10; 95\% CI: $0.48-2.54)$. None of the 8 women who used to smoke were a case, compared to 6 of the $108(5.5 \%)$ men.

In the reference laboratory, fluorescence microscopy was performed before the culture was initiated. Of the 127 patients who had three negative smears at the recruitment center, $104(81.9 \%)$ had also a negative smear at the reference laboratory. Of these, $13(12.5 \%)$ were culture positive. Twenty three patients who had three negative smears at the recruitment center had a positive smear at the reference laboratory and of these, 21 (91\%) were culture positive. 
Table 2. Characteristics of Cases and Controls

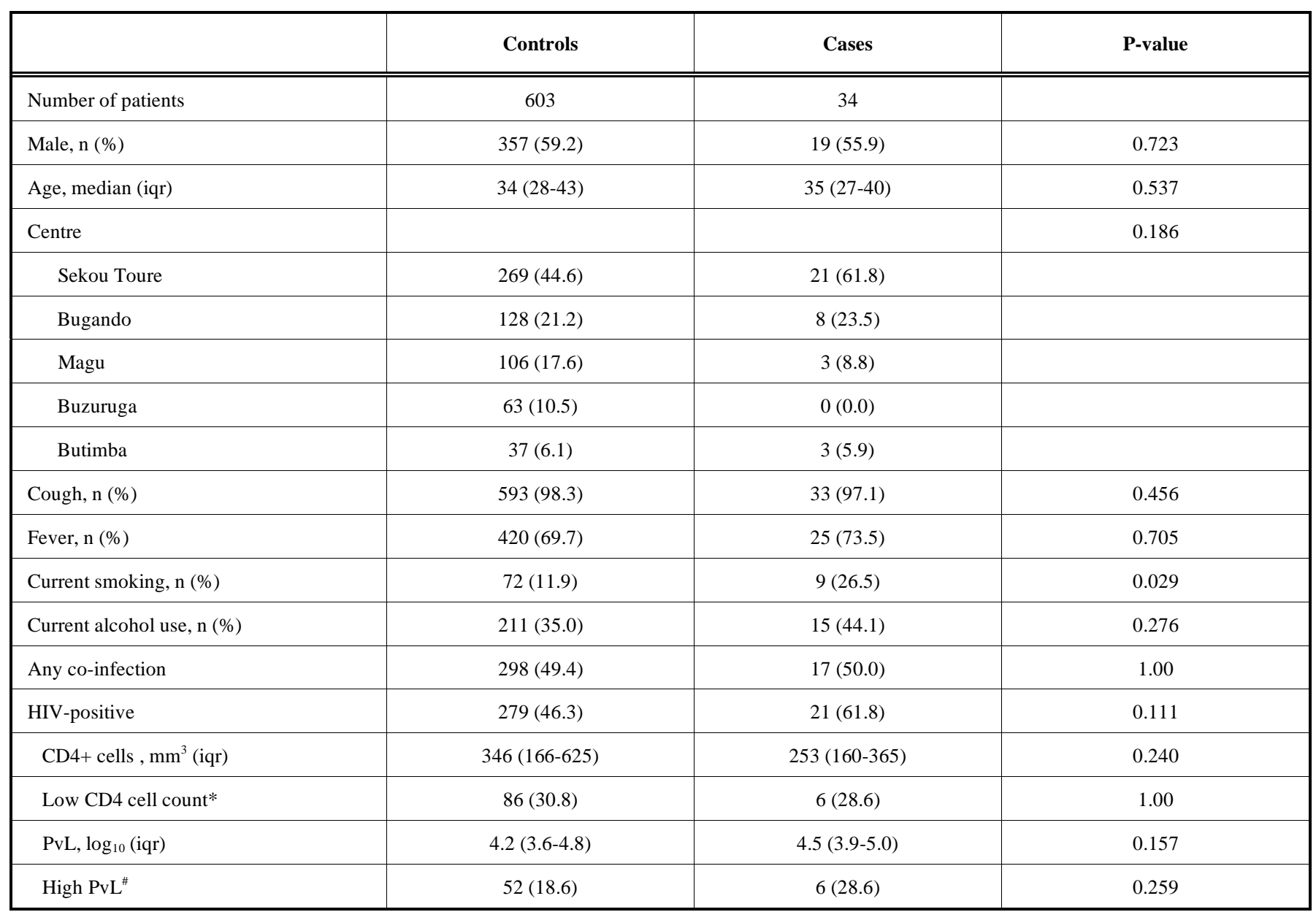

Iqr = inter-quantile range; Any co-infection = Malaria, S. mansoni, S. hematobium, A. lumbricoides, T. trichiura and S. stercoralis, HIV = Human Immunodeficiency virus; PvL = Plasma viral Load; * Low CD4 cell count $=<200$ cells $\mathrm{mm}^{3}$; ${ }^{*} \mathrm{High} \mathrm{PvL}=>5 \log _{10}$;

Table 3. Risk Factors for Being a Case

\begin{tabular}{|c|c|c|c|c|c|c|}
\hline & OR & $95 \% \mathrm{CI}^{\#}$ & $p$ & OR & $95 \% \mathrm{CI}^{\#}$ & $p$ \\
\hline Male & 0.934 & $0.57-1.56$ & 0.808 & & & \\
\hline Age, per year & 0.98 & $0.96-1.00$ & 0.184 & 0.97 & $0.95-1.00$ & 0.068 \\
\hline Alcohol use & 0.10 & $0.40-6.67$ & 0.488 & & & \\
\hline HIV-positive & 1.89 & $0.88-4.04$ & 0.101 & & & \\
\hline Smoking men & & & & 2.50 & $1.38-4.51$ & 0.002 \\
\hline Smoking women ${ }^{\S}$ & & & & 17.75 & $3.04-103.47$ & 0.001 \\
\hline
\end{tabular}

* In addition to sex and age; ${ }^{\#}$ based on robust standard error; ${ }^{\S}$ in comparison to non-smoking patients.

\section{DISCUSSION}

We found numerical differences in the percentage of TB patients with smear negative and culture positive results by diagnostic centers. This could possibly be due to laboratory quality services issues in terms of personnel qualifications and workload. A centre like Sekou Toure hospital and Bugando Medical Centre (BMC) had more patients recruited for the study and had more cases (smear negative but culture positive results). For program operational improvement it may be necessary to conduct AFB microscopy refresher 
training to the laboratory personnel to minimize the differences on quality of sputum smear microscopy. In addition, recruitment of additional staff to cope with a high workload could probably improve the quality of laboratory services. Laboratory monitoring and routine quality assurance should be reinforced. High workload and inadequate training of laboratory personnel in Tanzania have recently been described as factors related to a high proportion of falsenegative smears [17]. A similar finding was described in a study performed in Nairobi Kenya [18].

The study also showed that replacing routine culture by a negative confirmation smear seems not a viable option, as the 13 patients who were confirmed smear negative at the reference TB laboratory but still had a positive culture, would have been missed based on smear negative confirmation. With the possibility of increasing numbers of smear negative TB cases due to the HIV/AIDS epidemic, complementing smear negative results with culture seems to be a better option with respect to case detection.

Our study showed that current cigarette smoking was strongly associated with being a case (OR 3.61 95\% CI; 1.57-8.33), and that this association was stronger in women than in men. According to our knowledge, this is the first time that this association has been described. However, due to the very wide confidence intervals, the data can only serve as a first indication of a possible relationship between smoking and sputum smear results, and the possible modifying effect of sex on this relationship. Further studies that have an adequate number of patients must assess this relationship. If this relationship is indeed confirmed, modified diagnostic procedures for current smokers might be needed.

Smoking has been reported to be associated with an increased risk of developing TB disease [19], a slow healing process, delayed sputum conversion [7], and a high death rate [20].

The main limitation of our study is its post-hoc design. Result from such an analysis should be interpreted with care, since adequate measuring of confounding factors might not have been optimal. This design is also prone to selection bias. We have included all TB patients from the diagnostic centers who were confirmed cases through clinical suspicion, sputum examination and X-ray. Sputum culture was not part of the diagnostic work-up. Any misclassification of TBpatients is therefore non-differential with respect to culture result and would not have influenced the results of this study.

\section{CONCLUSION}

The frequencies of smear-negative culture-positive results differ between health facilities, indicating possible difference in quality of laboratory procedures. Strengthening of laboratory capacity is needed both for optimizing smear microscopy techniques, and for performing sputum cultures for diagnosing TB when there is a high rate of suspicion.

The TB program and other stakeholders should have collaborative efforts to control smoking. Messages on TB control to create public awareness should include strategies on the control of cigarette smoking to minimize its effect on TB. The association between smoking and smear negative culture positive TB needs to be assessed in adequately large studies.

\section{ACKNOWLEDGEMENTS}

The study was funded by the Danish International Development Assistance through the Council for Development Research and DBL Institute for Health Research and Development. The authors would like to thank all staff from health facilities, laboratories, and the National Institute for Medical Research, Mwanza Centre for their valuable contribution to the study, and the patients for participating. N. R., P. M., A. B. A. and H. F. developed the protocol. N. R. was responsible for conducting of the study and for data management, under supervision of P. M., A. B. A. and H. F. N. R and FvL. were responsible for data analysis and writing the first draft of the manuscript. All authors contributed to the final version of the manuscript. There were no conflicts of interest. The funding source had no influence on study design; collection, analysis and interpretation of data; in the writing of the report; and in the decision to submit the paper for publication.

\section{REFERENCES}

[1] World Health Organization Global Tuberculosis Programme 2006 WHO Report: Global Tuberculosis Control. World Health Organization, Geneva: Switzerland, 2006.

[2] Conde MB, Figueira CM, Moraes R, Fonseca LS, Deriemer K, Kritski AL. Predictive value of the acid fast smear for detection of Mycobacterium tuberculosis in respiratory specimens in a reference center Of HIV/AIDS, In: Rio De Janeiro, Ed., Brazil Mem: Inst Oswaldo Cruz 1999; 94: 787-90.

[3] Colebunders R, Bastian I. A review of the diagnosis and treatment of smear-negative pulmonary tuberculosis. Int $\mathbf{J}$ Tuberc Lung Dis 2000; 4: 97-107.

[4] Levy H, Feldman C, Sacho H, van der Meulen H, Kallenbach J, Koornhof H. A reevaluation of sputum microscopy and culture in the diagnosis of pulmonary tuberculosis. Chest 1989; 95: 1193-7.

[5] Brindle RJ, Nunn PP, Githui W, et al. Quantitative bacillary response to treatment in hiv-associated pulmonary tuberculosis. Am Rev Respir Dis 1993; 147: 958-61.

[6] Kanaya AM, David GV, Henry CF. Identifying pulmonary tuberculosis in patients with negative sputum smear results. Chest 2001; 120: $349-55$

[7] Gajalakshmi V, Peto R, Kanaka TS, Jha P. Smoking and mortality from tuberculosis and other diseases in India: retrospective study of 43000 adult male deaths and 35000 controls. Lancet 2003; 362: 507-15.

[8] Lienhardt C, Rowley J, Manneh K, et al. Factors affecting time delay to treatment in a tuberculosis control programme in a subsaharan african country: the experience of the Gambia. Int J Tuberc Lung Dis 2001; 5: 233-9.

[9] MacIntyre CR, Plant AJ, Hulls J, Streeton JA, Graham NM, Rouch GJ. High rate of transmission of tuberculosis in an office: impact of delayed diagnosis. Clin Infect Dis 1995; 21: 1170-4.

[10] Nyagosya R, Áse BA, Magnussen P, Mugomela A, Friis H. The effect of micronutrient supplementation on treatment outcome in patients with pulmonary tuberculosis: a randomized controlled trial in Mwanza, Tanzania. Trop Med Inter Health 2005; 10(9): 826-32.

[11] Bwire R, Borgdorff MW, Sticht-Groh V. Tuberculosis chemotherapy and sputum conversion among hiv-seropositive and hivseronegative patients in south-eastern uganda. East Afr Med J 1999; 76: 307-13.

[12] Githui WA, Kitui F, Juma ES, Obwana DO, Mwai J, Kwamanga D. A comparative study on the reliability of the fluorescence microscopy and ziehl-neelsen methods in diagnosis of pulmonary tuberculosis. East Afr Med J 1993; 70: 263-6.

[13] Heifets LB, Good RC. Current laboratory methods for the diagnosis of tuberculosis. In: Bloom BR, Ed., tuberculosis: pathogenesis, protection and control. 1994. American society for microbiology: Washington D.C. 2005.

[14] National Tuberculosis and Leprosy program manual Fifth ed. 2006. 
[15] WHO. Treatment Of Tuberculosis: Guidelines For National Programmes. Third ed. WHO/CDS/TB/2003.313. Geneva: WHO 2003.

[16] Range N, Magnussen P, Mugomela A, et al. HIV and parasitic coinfections in tuberculosis patients: a cross-sectional study in Mwanza, Tanzania. Ann Trop Med Parasitol 2007; 101 (4): 343-51.

[17] Basra D, Matee MIN, McNerney R. Quality assessment of sputum smear microscopy for detection of acid fast bacilli in peripheral health care facilities in Dar Es Salaam, Tanzania. East Afr Med J 2006; 83 (6): 36-10
[18] Hawken, Under-diagnosis of smear-positive pulmonary tuberculosis in Nairobi, Kenya. Int J Tuberc Lung Dis 2001; 5: 360-3.

[19] Gupta KP, Gupta R. Association between smoking and tuberculosis. Indian J Tuberc 2003; 50: 5-7.

[20] Hassmiller KM. The association between smoking and tuberculosis. Salud Public Mex 2006; 48(Suppl 1): S201-S216.

(C) Range et al.; Licensee Bentham Open.

This is an open access article licensed under the terms of the Creative Commons Attribution Non-Commercial License (http://creativecommons.org/licenses/ by-nc/3.0/) which permits unrestricted, non-commercial use, distribution and reproduction in any medium, provided the work is properly cited. 